\title{
The evaluation of buildings energy consumption and the optimization of district heating networks: a GIS-based model
}

\author{
Chiara Delmastro • Guglielmina Mutani • \\ Laura Schranz
}

Received: 31 July 2014/ Accepted: 31 December 2014/Published online: 27 January 2015

(C) The Author(s) 2015. This article is published with open access at Springerlink.com

\begin{abstract}
The European buildings occupy a key place among the major energy consumer sectors, with high savings potential. The development of urban planning tools helpful to understand the right policy strategies turning the settled sustainable targets into real energy consumption savings is now a real challenge. Into this paper is described a methodology, for the mid-long term scenarios analysis, able to asses the buildings energy consumption of a municipality by means of a simulation approach and of a geo-referenced characterization of the stock. A thermal model, based on real consumption data, has been used to evaluate space heating energy demand; different savings opportunities have been simulated. Moreover, from the geo-referenced representation of the district heating network, through the integrated procedure, it is possible to perform the optimization of the network layout. A case study application in Turin is presented. Main results are the evaluation of energy consumptions, total costs of the interventions and the release of policy suggestions. Thanks to geo-referenced maps is allowed to put in evidence criticalities and policy effects through thematic maps. The methodology highlights the advantages of coupling a geographical information system application and energy demand forecasting model to build up a tool aimed at supporting decision-making.
\end{abstract}

Keywords Energy consumption - Residential buildings · District heating · Optimization · GIS

Published in the Special Issue "8th AIGE Conference (Italian Association for Energy Management)".

C. Delmastro - G. Mutani - L. Schranz $(\bowtie)$

DENERG, Politecnico di Torino, Corso Duca degli Abruzzi, 24, 10129 Turin, Italy

e-mail: laura.schranz@polito.it

\section{Introduction}

Nowadays in Europe, the building sector is one of the most energy consumers: the individuation of suitable measures to reach the fixed reduction target for 2020 is a real challenge for policy makers.

Consequently, great attention is now focused into the planning tools for the forecasting of the effects of different interventions and measures. For the analysis of the energy systems of a Country or a region and, less frequently, of a local system like a metropolitan area, energy models like those of the MARKAL-TIMES [1] and MESSAGE [2] families have been (and are) widely used. Such models are a technology-rich tool for evaluating long-term trajectories of multi-regional energy systems.

Together with the diffusion of geographic information systems (GIS) applications, the growing interest in local energy planning and the increase of the power of the computing tools have encouraged the development of new exercises in the field [3].

The integration of space variable is a skill necessary in almost every energy planning process; in fact, it is helpful to assist the siting of new generation facilities, to determine the optimum route for new distribution and transmission network lines, and to develop emergency evacuation plans around facilities.

The energy planning process of an urban settlements or a district first require defining its reference energy system: to evaluate the energy demand by end use and by fuel, to identify the existing end-use technologies and to describe the local supply plants and infrastructures. Thanks to the GIS tool, all these data are the geo-referenced.

The presented paper is focused on the retrofit of buildings and the refurbishment of the district heating network in the Centro Residenziale Europa area of Turin-CRE 
(with a gross heated volume of about $650,000 \mathrm{~m}^{3}$ ). The analysis has been performed using a specific GIS-integrated modeling tool able to identify and characterize a suitable mix of measures/retrofit/technologies/infrastructures in the direction of a "smart" and sustainable built environment.

Three scenarios are proposed: Baseline, Medium and Advanced. In all the scenarios, the renovation of the district heating network is included; the difference among them is related to the level of buildings retrofit. Moreover, for that exercise only water heating and space heating services have been taken into account. In Italy, typically, these services are supplied by individual heating systems, using boilers (gas, oil, solid fuels) or electric heating system; contrarily, district heating is widely adopted in Turin.

The CRE district, built from 1968 to 1974, is sited near to the Fiat Mirafiori factory, in the South-West of the city, between Tazzoli, Orbassano and Reni axes.

The new prefabrication system, produced by the UPIR factory and very diffused at that period, made available elegant apartments type at lower price in comparison to other contemporary private constructions. The resulting district, is characterized by blocks of flats-eleven floors tall-arranged in a $U$ around the border of the affected area.

After more than 40 years, the Centro Residenziale Europa district heating (DH) network is quite old and characterised by high heat losses that justify the desire of the inhabitants to promote a refurbishment.

\section{Materials and methods}

The first step of the procedure is the collection of the Base Year (2010) data: global domestic product (GDP), population, floor area, per capita income, energy use, energy policies, base maps for the GIS analysis. Moreover, it is necessary to describe the building stock (construction period, shape factor...)-identification of reference buildings-and to characterize the district heating network (existing and planned). Using the GIS software, all the buildings and the networks are geo-referenced. It allows the creation of a detailed representative GIS-Database with the characteristics of the buildings and their end-use technologies (efficiency and life) [4] (Fig. 1).

GIS data could be open source data, free data made available by private companies, fee-based data sources; they are available from many sources including federal, state, and local government database.

Through an embedded procedure of the Model, in absence of existing local network or in case of the refurbishment of the existing one, it is possible to find (under suitable user-defined constraints) the path that minimizes the total cost. This procedure is the core of the project; it starts by use, for each geo-referenced building, a thermal model to evaluate the space heating and hot water energy demands.

Then, for all the buildings, using bottom-up models, it is possible to describe and to project a certain number of reference processes supplied by a series of commodities (coal, natural and city gases, LPG, dh vectors, electricity, etc.) to provide the scenarios analysis.

The GIS application is present in all these phases, in fact:

(i) It manages the input of the spatial data

(ii) It transfers data to tables, plots and maps on the urban (over- and under-ground) space the outputs of the runs ("smart" indicators, energy and emission maps, infrastructure layout) induced by the policies and actions assumed or suggested by the model itself.

The case study

A case study concerned with the CRE area in the municipality of Torino was carried out. Torino is located in the temperate continental climatic zone. Into the examined area, a new DH system is going to supply heat (both for space heating and water heating) to 14 buildings. The area is characterised by three building types with towers, multifamily apartment buildings and low-rise row buildings and two construction periods from 1971-1980 to 1881-1990. Some units of the same buildings complex have different $\mathrm{DH}$ heat exchangers and they have been considered as a single unit and, for that reason, the total number of analysed buildings grows to 21 (Fig. 2).

\section{Heating requirements}

This section looks at the heating requirements, dictated by both space heating and domestic hot water production, in the CRE district. The objective is to evaluate the peak thermal power by each building.

Turin is one of the most district-heated cities in Europe with a heated volume of about $54 \mathrm{Mm}^{3}$. During the heating season 2010-2011, energy consumption data for about three hundred residential buildings (with different geometric characteristics and building types) were collected thanks to the cooperation with the company managing district heating in Turin [5]. From these data, deriving from the heat metering of 288 buildings, an energy consumption model has been evaluated and applied on the Centro Residenziale Europa as shown in Table 1.

From literature, the energy consumption for heated spaces principally depends on: climatic data, period of 
Fig. 1 Methodological approach

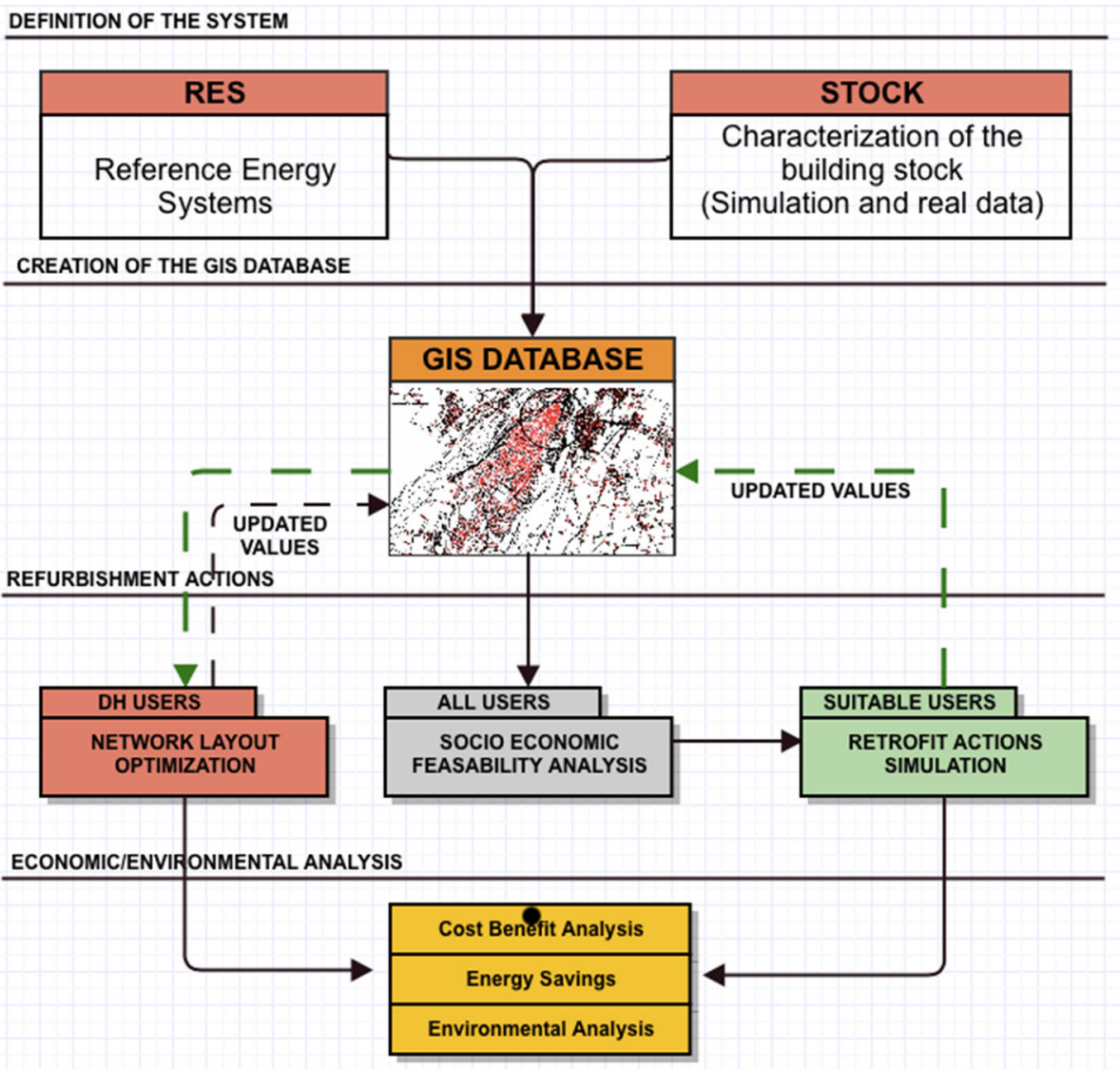

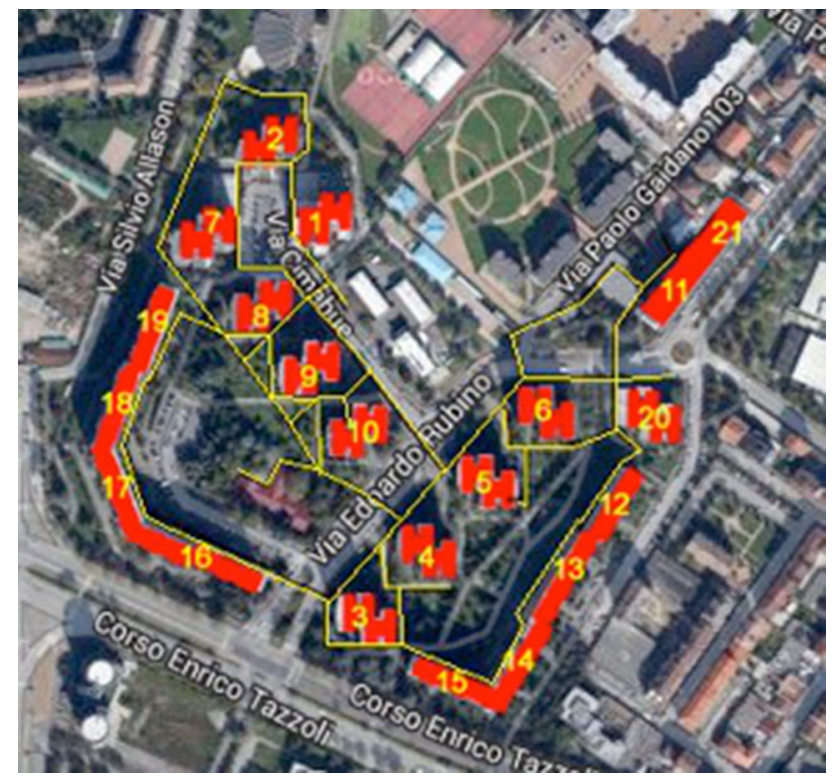

Fig. 2 Building stock viewed with a GIS tool

building's construction, building's compactness or surface to volume ratio and percentage of the buildings' heated volume [6-11].
For residential buildings, energy is dominated by heating demand and increasing compactness and buildings' density, heat loss is generally reduced even if the availability of solar heat gains also influences energy consumption [12].

In this work, energy consumption on existing buildings depends by the degree days (used in this model to normalise the energy consumption on climate data), by the envelope's thermal insulation (related to the period of construction) and by the geometrical shape of buildings. This simplification does not take into account important factors such as: solar gains, the variability of the indoor air temperature and the renovation works to improve energy efficiency that may have changed the energy consumption. On the other hand, only a simplified model can be used on urban scale to evaluate energy demand for a municipality, province or region with a GIS tool.

For the domestic hot water production, the net energy requirement was considered equal to $17.05 \mathrm{kWh} /\left(\mathrm{m}^{2} \mathrm{a}\right)$ as average value proposed by the UNI/TS 11300-2:2008; for the evaluation of primary energy has been chosen a performance of plants depending by the construction period: before 1976 of about $40 \%$, from 1976 to 2005 of 60 and 
Table 1 Energy consumption for space heating $\left(\mathrm{kWh} / \mathrm{m}^{2} / \mathrm{y}\right)$ of residential buildings in Turin as function of surface to volume ratio and construction period (weather data from Italian Standard UNI 10349:1994, 2,617 degree-days)

\begin{tabular}{lll}
\hline $\begin{array}{l}\text { Building } \\
\text { construction } \\
\text { period }\end{array}$ & $\begin{array}{l}\text { Primary energy for } \\
\text { space heating } \\
{\left[\mathrm{kWh} /\left(\mathrm{m}^{2} \mathrm{a}\right)\right]}\end{array}$ & $\begin{array}{l}\text { Coefficient of } \\
\text { determination }\end{array}$ \\
\hline$<1919$ & $\mathrm{y}=133.28 \times \mathrm{S} / \mathrm{V}+102.58$ & $\mathrm{R}^{2}=0.76$ \\
$1919-1945$ & $\mathrm{y}=123.59 \times \mathrm{S} / \mathrm{V}+99.70$ & $\mathrm{R}^{2}=0.77$ \\
$1946-1960$ & $\mathrm{y}=93.30 \times \mathrm{S} / \mathrm{V}+97.22$ & $\mathrm{R}^{2}=0.80$ \\
$1961-1970$ & $\mathrm{y}=91.05 \times \mathrm{S} / \mathrm{V}+96.51$ & $\mathrm{R}^{2}=0.76$ \\
$1971-1980$ & $\mathrm{y}=91.95 \times \mathrm{S} / \mathrm{V}+68.92$ & $\mathrm{R}^{2}=0.76$ \\
$1981-1990$ & $\mathrm{y}=80.76 \times \mathrm{S} / \mathrm{V}+71.82$ & $\mathrm{R}^{2}=0.79$ \\
$1991-2005$ & $\mathrm{y}=71.26 \times \mathrm{S} / \mathrm{V}+72.24$ & $\mathrm{R}^{2}=0.95$ \\
\hline
\end{tabular}

$70 \%$ after 2005 . These values emerge from a comparison of the Italian state of the art [13].

The energy consumption model has been calibrated with real energy consumption data, external temperatures and an average value of percentage for the heated volume in buildings blocks. Then, this model is valid only on a specific urban form, with specific building's archetypes and envelopes, occupant behavior, thus for a specific town.

Results of this analysis are represented in Table 2; relative differences between consumption data are below
$11 \%$ so the heated volume considered and the characteristics of heating systems and building envelope are correct.

The feasibility of renovation works

This section describes the methodology to define the feasibility of renovation works for residential buildings in Turin. The energy saving improvements on buildings can be considered only if the socio-economic conditions of inhabitants can support them.

Socio-economic data related to population and buildings census ISTAT of 2001 [14] (more recent data are not yet available) have been used to calculate the feasibility of renovation works. The methodology that has been developed is intended to be easily replicable even in other territorial contexts, and allows a progressive update and improvement of the data.

For the purposes of determining an indicator to evaluate the feasibility of renovation works in residential buildings, the following socio-economic factors were selected:

1. The age factor $\left(f_{a}\right)$ : calculated by dividing the population included in the age group between 25 and 69 years old for the total resident population.

Table 2 Volume, heat peak power and calculated plus measured specific energy consumption

\begin{tabular}{|c|c|c|c|c|}
\hline Building ID & Heated volume $\left(\mathrm{m}^{3}\right)$ & Specific peak power $\left(\mathrm{W} / \mathrm{m}^{3}\right)$ & Calc. consumptions $\left(\mathrm{kWh} / \mathrm{m}^{2} / \mathrm{y}\right)$ & Real consumption $\left(\mathrm{kWh} / \mathrm{m}^{2} / \mathrm{y}\right)$ \\
\hline 1 & 26,265 & 30.5 & 111.67 & 116.88 \\
\hline 2 & 25,790 & 27.1 & 113.69 & 110.96 \\
\hline 3 & 25,127 & 27.9 & 118.88 & 107.84 \\
\hline 4 & 25,127 & 27.9 & 118.88 & 114.66 \\
\hline 5 & 24,402 & 28.7 & 119.02 & 128.3 \\
\hline 6 & 25,377 & 27.6 & 118.58 & 116.99 \\
\hline 7 & 25,084 & 27.9 & 116.73 & 130.08 \\
\hline 8 & 25,084 & 27.9 & 115.73 & 110.05 \\
\hline 9 & 25,645 & 27.3 & 115.58 & 113.73 \\
\hline 10 & 25,349 & 27.6 & 115.63 & 109.5 \\
\hline 11 & 13,528 & 44.8 & 145.04 & 162.79 \\
\hline 12 & 21,669 & 28.8 & 115.11 & 115.92 \\
\hline 13 & 28,624 & 29.3 & 114.26 & 117.58 \\
\hline 14 & 21,051 & 29.8 & 113.56 & 119.61 \\
\hline 15 & 30,571 & 29.8 & 113.71 & 119.83 \\
\hline 16 & 22,108 & 23.9 & 118.94 & 108.65 \\
\hline 17 & 34,687 & 25.1 & 108.47 & 113.86 \\
\hline 18 & 32,337 & 24.6 & 109.46 & 111.68 \\
\hline 19 & 24,396 & 24.8 & 116.45 & 112.32 \\
\hline 20 & 25,704 & 27.2 & 118.63 & 114.98 \\
\hline 21 & 2,164 & 25.0 & 169.11 & 189.82 \\
\hline
\end{tabular}


2. The employment factor $\left(\mathrm{f}_{\mathrm{e}}\right)$ : calculated by dividing the total of the workers for the resident population of working age (15-64 years).

3. The property factor $\left(f_{p}\right)$ : calculated by dividing the number of occupied property dwellings for the total number of occupied dwellings.

4. The family factor $\left(\mathrm{f}_{\mathrm{f}}\right)$ : calculated by dividing the number of families with more than three components to the total number of families.

Higher values of these factors mean a growing socioeconomic feasibility of the renovation works on buildings.

Each of the four socio-economic factors contributes to the formulation of a final indicator (Eq. 1):

$F=\operatorname{function}\left(f_{a}, f_{e}, f_{p}, f_{f}\right)$.

However, each factor takes a different weight according to its importance in relation to the objective of the analysis; thus, it is necessary to define the weights of the different factors to get an overall indicator of socioeconomic feasibility expressed by a value between 0 and 1 .

Prior to the allocation of weights, the normalization of the values of the five factors with respect to their mean value was completed. In fact, each factor has an average value, which depends substantially on the size of the described variable.

As represented in Table 3, for example, the age factor has an average value of 0.76 , while for the family factor it is equal to 0.33 . The normalization of the four factors is carried out to have a mean value of 0.5 for all the variables multiplying the value of the various factors by $0.5 /$ (average value); in this way all variables have the same influence on the feasibility of renovation works.

The weights assigned to the four factors are shown in Table 3; the choice of the weights has been made in such a way that their sum is equal to one, with the maximum weight attributed to the employment factor, while to the age factor has been assigned less weight.

The assignment of a weight to each factor made it possible to calculate a final indicator of feasibility $F$, obtained by adding the normalized four socio-economic factors each multiplied by their relative weight (Eq. 2):

$F=0.15 \cdot f_{a}+0.35 \cdot f_{e}+0.30 \cdot f_{p}+0.20 \cdot f_{f}$

Table 3 Mean values and weights for the four socio-economic factors of the census sections of the municipality of Turin

\begin{tabular}{lllll}
\hline Factors & $\mathrm{fa}$ & $\mathrm{fe}$ & $\mathrm{fp}$ & $\mathrm{ff}$ \\
\hline Average value & 0.76 & 0.54 & 0.87 & 0.33 \\
Weigh & 0.15 & 0.35 & 0.30 & 0.20 \\
\hline
\end{tabular}

Considering all the census sections of the municipality of Turin, the feasibility index $F$ has a mean value of 0.5 and a standard deviation of 0.14 .

Using the mean value and the standard deviation of the indicator feasibility $F$, four classes of feasibility were built which allowed assigning different levels of energy retrofitting works [15]:

- Class 1. From zero to the average value minus the standard deviation.

- Class 2. Lying between the mean value minus the standard deviation and the mean value.

- Class 3. Lying between the mean value and the mean plus the standard deviation.

- Class 4. Higher than the mean value plus the standard deviation.

In the CRE district, there are four census sections with the buildings in Class 3 and 4; the possibility to make renovation works on the buildings to increase energy efficiency is medium-high.

\section{The potential energy savings}

This paragraph refers to the evaluation of the possible energy savings obtainable by retrofit interventions on the area. Lower heat losses mean that the building heat design load will be smaller. This could offer opportunities to reduce the pipe diameter and consequently to reduce the capital investment required for the distribution network and, potentially, the investment costs (smaller trenches, excavation and refilling costs). Usually, the heat network is the highest capital cost element particularly in low-density developments.

The identification of the four classes described in the previous section, allows identifying a level of renovation works depending on the socio-economic characteristics identified.

From the analysis made by the ENEA for the Piedmont Region [16] results that between all the possible energy savings interventions, $63 \%$ of them is related to the replacement of windows. It was therefore decided to start with the replacement of windows for the lower classes (Medium "M" scenario), then progress with the thermal insulation of the roof and the floor below the building for Class 3. Finally, all the actions of the previous classes have been considered, plus the insulation of the walls for Class 4. These last two scenarios are called Advanced "A".

The energy savings resulting from renovation works on the buildings of Turin were made by reference to the actual consumption data and calculations on some buildings-type chosen for the different periods of construction and different surface to volume ratio [5, 17] (Fig. 3). 
Fig. 3 Obtainable energy savings $(\mathrm{kWh} / \mathrm{y})$ for the 21 buildings

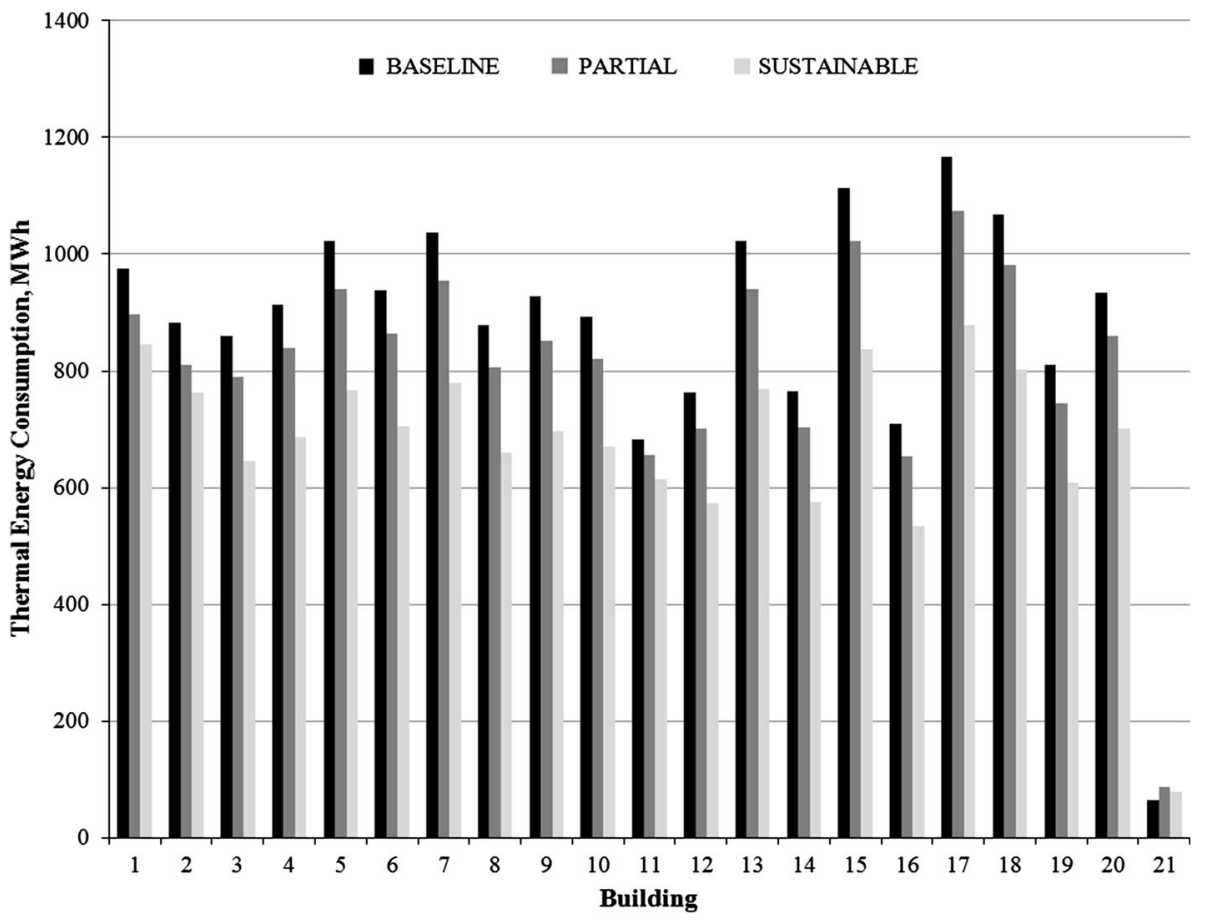

From the Baseline scenario, with the "Medium" the obtained energy savings increases of about $4 \%$ and with the "Advanced" of $38 \%$. Considering a heat cost for the district heating in Turin of $0.061 € / \mathrm{kWh}$, with the Medium scenarios the energy saving is of about $182,000 € / y$, while with the Advanced Scenario 525,000 €/y.

The district heating network

In this paragraph, it is explained the procedure adopted for the optimization of the district network path. The determination of the optimum configurations and pipe-dimensioning of a DH system within an urban area would involve all the elements which constitute this system: source plants (site, size, type and fuel supply) and network layout.

In this analysis, the CRE network already exists and needs to be refurbished because the heat losses are higher that $7 \%$. The substation is not going to be changed, consequently the "source analysis siting and dimensioning part" can be neglected.

The geo-referenced building stock attribute tables are imported in Matlab. The useful data (users coordinates, users peak power, source position, network branches and nodes position, etc.) are collected and used for the network optimization analysis.

The optimization procedure is based on the Computer Aided Procedure for Local Energy Planning (CAPLEP) procedure [3]: with reference to the graph of the existing urban structure/street connections (Fig. 2), constituted by nodes and branches where users and source play the role of principal nodes; the proposed algorithm searches for the tree- belonging to the graph- representing the optimum connection.

The algorithm on which the procedure is based is referred to the Steiner algorithm, typically used for electric grid. Since for a district heating network the shortest path does not permit the identification of the lowest cost, a parameter to be multiplied for the branches lengths, called "Importance of i-th branch", has been introduced into the procedure: it associates a cost and urban constrains information to all the branches.

This "Importance" parameter (I) is constituted by three factors depending on: the source position, the peak power of users and the urban constrains (Eq. 3):

$I(i)=[1 / P(i)]^{\alpha} \cdot S(i)^{\beta} \cdot C U(i)$.

1. The source factor $(\mathrm{S})$ is going to "weigh" each branch in relationship to the distance between the source plant and the i-th branch (middle point).

2. The users peak power factor (P) is the summation of the contribution of each user peak power on the i-th branch, depending on the distances between the users and the i-th branch itself: for each consumer, a normal distribution of "weight" depending on heat peak power and distances is created.

3. Urban constrains are considered thanks to the $\mathrm{CU}$ factor: it gives information about the suitability of a branch to be crossed by the district heating network. 
If $\mathrm{CU}$ is equal to zero it means that it is not allowed to run through that $\mathrm{i}$-th branch (for example if physical barriers are present) and that the i-th branch is automatically excluded from the base graph-its equivalent length is assumed equal to zero to reduce the algorithm's computational cost. If no obstacles are present on the i-th branch $\mathrm{CU}$ is equal to one and the branch is considered into the initial graph.

4. Exponents alfa $(\alpha)$ and beta $(\beta)$ weighs the importance, respectively of the inverse of the users peak power factor $[1 / \mathrm{P}(\mathrm{i})]$ and of the source factor $(\mathrm{S})$. They depend on the building spatial distribution and district geometry; they are evaluated by an iterative procedure.

Obviously, also the lengths of the branches are affecting the cost of the pipes, consequently, the "cost-equivalent branch length" is evaluated (Eq. 4):

$L_{e q}(i)=L(i) \cdot I(i)$

where:

- Leq: new equivalent length of the i-th branch, modified according to investment cost;

- L: real length of the i-th branch;

- I: importance factor.

The output table obtained by the program includes the ID number of the "selected" branches. Following the layout path definition, computations typical of thermo-economical analysis [18, 19] are carried out separately on each pipe branch to evaluate: pipes' flow, diameter, velocity, pressure difference, investment cost and, at the end, the cost of the network. In the costevaluation part only investment cost has been considered: operational and maintenance cost have been neglected in this first part of the planning analysis. Moreover, a feasibility analysis based on minimum and maximum acceptable velocities and pressure differences analysis is performed.

The outcome of the optimization consists in a tree network that lies very near to the optimum; anyway must be considered that final adjustment can still be made, in fact, the reliability of the network does not always permit a simple tree structure. Furthermore, global strategies for the growth of DH networks will play a decisive role (including in the dimensioning all the "potential users").

For this analysis, future network extension is assumed not to be required and the network has been dimensioned in accordance with the Piedmont Region catalog of commercially available twin pipes. The Matlab table obtained by the optimization process is than re-imported into the

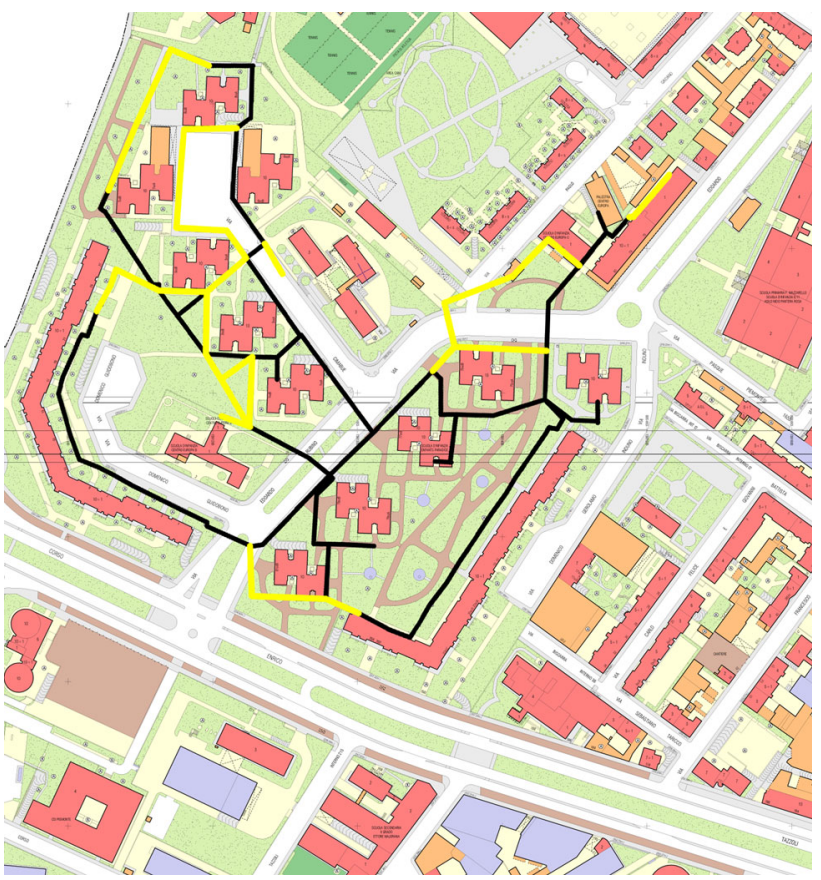

Fig. 4 District heating network layout

GIS tool, allowing to update existing data, to add the new ones and to create the map.

Starting from the consumers peak heat demand of 14 , $9 \mathrm{MW}$ and a $30{ }^{\circ} \mathrm{C}$ temperature gradient among inlet and outlet pipes, it results a piping network with a total length of about $1.73 \mathrm{~km}$ in the layout of the branched type-the length of the end-user connections is not being included (Fig. 4).

In Fig. 4 is it possible to observe the GIS output map. The darker branches represent the optimum result. Main outcomes are:

- Network length (m): 1,729;

- DN Max (mm): 200;

- $\mathrm{V} \max (\mathrm{m} / \mathrm{s}): 2.68$;

- Economic savings due to heat losses reduction $(\mathrm{k} € / \mathrm{y})$ : 70;

- Pipeline investment cost (k€): 86;

- Total network investment cost $(\mathrm{k} €): 232$;

- Pay-back period of the intervention (y): 3 .

The network found by a plain Steiner algorithm (without weighting the branches) is characterized by a total investment cost of $237 \mathrm{k} €$ and a length of $1,670 \mathrm{~m}$; these outcomes are not so different with respect to the results obtained with the presented methodology. This is due to the low number of buildings and branches involved in the analysis of the CRE refurbishment, but in wider networks the difference can be significant. 


\section{Results}

Last, an evaluation from the user's economic point of view is provided for the three intervention scenarios with different feasibility indexes: the Baseline (just district heating network is refurbished), the Advanced (district heating refurbishment and complete building retrofit) and the Medium (district heating refurbishment and windows substitution). From the distribution company's point of view an interesting evaluation is the energy-saving costs associated to the refurbishment of the heat network.

Since for all the interventions the district heating network refurbishment is provided, the cost-benefit analysis is referred to the choice between the Medium and the Advanced scenarios and to the identification of the pay back periods with respect to the Baseline case.

The investment costs are referred to the medium Italian costs collected by ENEA: $330 € / \mathrm{m}^{2}$ for the refurbishment of windows, $55 € / \mathrm{m}^{2}$ for the thermal insulation of the vertical facade and $33 € / \mathrm{m}^{2}$ for insulation of heat dispersant slabs. Investments are supposed to be paid in 10 years with an interest rate of $5 \%$ and the $65 \%$ State energy efficiency incentives are supposed to be used. For energy savings related costs it has been used the heat price applied by IREN Mercato S.p.A. in Turin and the annual growth rate of energy price supposed to be $6 \%$ while maintenance costs are supposed to be $4 \%$ of investment costs.

Using these assumptions it results a pay-back period of about 10 years for the Advanced scenario and of about 13 years for the Medium one (Fig. 5). Considering that only $50 \%$ of buildings can renovate in a short time period, the pay-back time is much higher: of about 16 years for the Advanced scenario and more than 20 years for the Medium one.

The result highlights that even if the Medium scenario (substitution of windows) is the most diffused and simple, it is not the most convenient and effective with pay back period more than 1/3 higher. Important further evaluations could be connected to the combination of refurbishment interventions and renewable energy sources application, especially for solar thermal water heating and PV systems.

\section{Discussion}

In this paper, a model to evaluate the heating demand of a set of buildings located in the Centro Residenziale Europa of Turin is defined, starting from real consumption data. The geo-referenced representation of the building stock with the GIS tool consents to distribute with detail the energy consumption, the emissions and the energy savings potential.

Furthermore, using the GIS representation of the original streets graph and through a cost-optimization procedure, it has been evaluated the best way to renovate the existing district heating network, supposing not to change the power plant site and typology. Combining the heat distribution network retrofit with savings energy interventions on buildings' facades, three different scenarios with different feasibility indexes have been analysed: Baseline (DH refurbishment), Medium (DH refurbishment plus windows retrofit) and Advanced (DH refurbishment and a complete retrofit).

Fig. 5 Pay-back periods

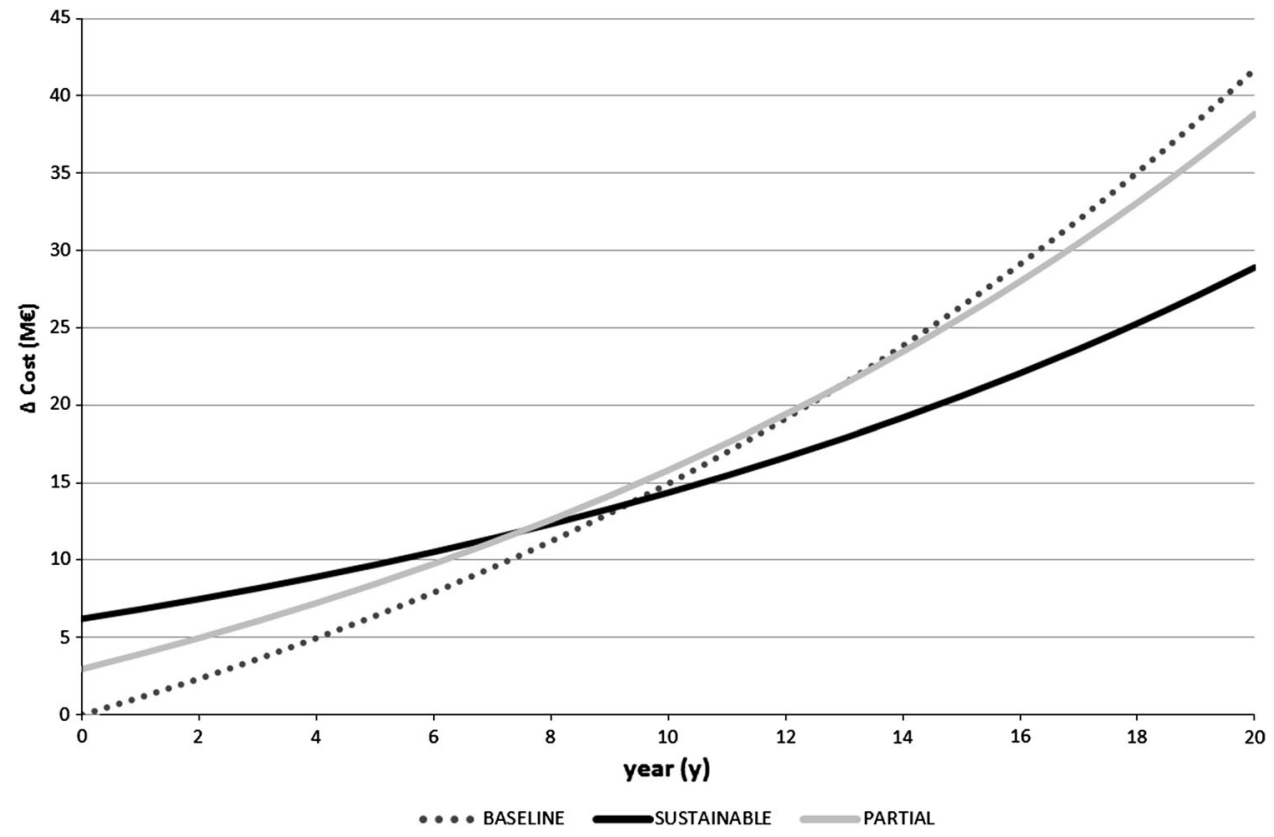


The energy savings scenarios will reduce the peak heat power demand with a reduction in term of diameters of the distribution network and of the cost of the generator. So energy efficiency interventions on buildings should be done before the network renovation.

The "Advanced" scenario, as expected, results economically more appealing with respect to the "Medium" one, even if the solution of the windows substitution is the most diffused and simple to adopt.

An interesting consideration is that the Italian peak power demand evaluations, for the Turin climatic zone (E), are referred to a project temperature of $-8{ }^{\circ} \mathrm{C}$, average of minimum yearly temperature on 20 years excluding the absolute minimums. During last years, taking into account global warming effect, the minimum yearly temperatures grew of $2{ }^{\circ} \mathrm{C}$ to an average of about $-6{ }^{\circ} \mathrm{C}$, corresponding to $7 \%$ of peak power reduction for the plant dimensioning. Excluding that in this analysis the source power plant already exists, a $7 \%$ peak power reduction is not enough to reach a lower commercially available power level reducing the investment cost of the boiler. This evaluation will be investigated in future researches on bigger networks.

Finally, this methodology seems to be very useful to plan energy savings measures on urban scale, considering the influence of each building heat demand on the heat network distribution with the optimization of the network. It must be specified that the applied methodology, using GIS tool on an urban scale, could be affected by the availability and quality of data.

Acknowledgments The authors would like to express their deep gratitude to Eng. Sonia Bertocci, for her help in collecting the data about district heating consumption.

Open Access This article is distributed under the terms of the Creative Commons Attribution License which permits any use, distribution, and reproduction in any medium, provided the original author(s) and the source are credited.

\section{References}

1. Tosato, G.C.: Introduction to ETSAP and the MARKAL-TIMES models generators. International Energy Agency: NEET Workshop on Energy Technology Collaboration (2008)

2. International Institute for Applied System Analysis (IIASA): http:// www.iiasa.ac.at/web/home/research/researchPrograms/Energy/ MESSAGE-model-regions.en.html
3. Lavagno, E.: Advanced Local Energy Planning (ALEP): a Guidebook, Annex 33 of the Energy Conservation in Buildings and Community Systems Program, KEA-B.W., DEU (2000)

4. Ascione, F., De Masi, R.F., De Rossi, F., Fistola, R., Sasso, M., Vanoli, G.P.: Analysis and diagnosis of the energy performance of buildings and districts: Methodology, validation and development of Urban Energy Maps, pp. 270-283, Cities (35) (2013)

5. Mutani, G., Pairona, M.: A model to evaluate the heating energy consumption for residential buildings in Turin, Ed. Maggioli Journal: L'Ufficio Tecnico, pp. 21-36, (2014)

6. AA.VV.: Cities and energy. Urban Morphology and Heat Energy Demand, Final Report, LSE Cities Research Team and EIFER Research Team, London (2014)

7. Ratti, C., Raydan, D., Steemers, K.: Building form and environmental performance: archetypes, analysis and an arid climate, pp. 49-59, Energy and Buildings (35) (2003)

8. Rode, P., Kiim, C., Robazza, G., Viejo, P., Schofield, J.: Cities and energy: urban morphology and residential heat-energy demand, pp. 138-162, Environment and Planning B: Planning and Design 2014 (41) (2014)

9. Salat, S.: Cities and Forms: On Sustainable Urbanism, CSTB Urban Morphology Laboratory Ed (2011)

10. AA.VV.: Cities and Energy. Urban Morphology and Heat Energy Demand, Report, LSE Cities Research Team, London (2012)

11. Mitchell, G.: Urban Development, Form and Energy Use in Buildings: A Review for the SOLUTIONS Project. Draft Paper, EPSRC SUE SOLUTIONS Consortium (2005)

12. Miller, N.: Urban form and building energy: quantifying relationships using a multi-scale approach, Thesis in the Faculty of Resource Management and Environmental Studies, University of British Columbia (Vancouver) (2013)

13. Fracastoro, G.V., Serraino, M.: A methodology for assessing the energy performance of large scale building stoks and possible applications, pp. 844-852, Energy and Buildings (43) (2011)

14. Italian National Institute of Statistics ISTAT, www.istat.it/en/

15. Vicentini, G., Mutani, G.: Boosting energy efficiency and RES in urban contexts: from the plan to the project, Smart City Expo World Congress, Barcelona, November 18-20th, in press (2014)

16. ENEA, Le detrazioni fiscali del $55 \%$ per la riqualificazione energetica del patrimonio edilizio esistente-2012, Italian National Agency for New Technologies, Energy and Sustainable Economic Development (ENEA), ISBN 978-88-8286-301-2 (2014)

17. Mutani, G., Vicentini, G.: The open data for the analysis of primary energy demand of the residential buildings and the potential energy savings, pp. 15-37, Smart City Exhibition, Bologna (2013)

18. Frangopoulos, C.A.: Thermoeconomic Functional Analysis and Optimization. Energy, pp. 563-571, The International Journal, (12, No. 7), (1987)

19. Chinese, D.: Optimal size and layout planning for district heating and cooling networks with distributed generation options, pp. 385-419, International Journal of Energy Sector Management 2(3) (2008) 